\title{
23. PACULTY OF
}

\section{FPM response to concern related to the safety of steroids injected as part of pain procedures during the current COVID-19 virus pandemic}

\section{Introduction}

Members have asked questions as to the safety of use of injected steroids for pain procedures during the current COVID-19virus pandemic.

Steroid use is common in pain procedures with the aim of easing pain, increasing mobility and quality of life. Their duration of effect is variable but can provide several months of benefit. The immunological impact of steroids given this way in patients with COVID-19 is unknown. As a result of the long quarantine period of an average of 14 days, there is also a risk that asymptomatic patients who are carrying the vi rus could be treated, potentially putting them at increased risk of an adverse outcome from the virus. There does appear to be higher risk patient groups, notably the elderly and those with comorbidities resulting in higher mortality rates.

Moreover, one must consider the risk of admitting patients for elective procedures in a hospital setting where they may be exposed to patients being acutely treated for COVID-19infection or other illnesses. There is also the risk of hospital staff being exposed to potentially infected patients.

\section{Use of steroids in treatment of COVID-19}

The current WHO guidance ${ }^{1}$ for the management of severe acute respiratory infection in patients with COVID-19 is not to routinely give systemic corticosteroids unless indicated for another reason. This is because steroids have been associated with an increased risk for mortalityin patients with influenza and delayed viral clearance in patients with Middle East respiratory syndrome coronavirus (MERS-CoV) infection. Although they were widely used in management of severe acute respiratory sy ndrome (SARS), there was no good evidence for benefit, and there was persuasive evidence of adverse short- and long-term harm ${ }^{2}$. A recent study of patients with COVID from China, reports that patients receiving corticosteroids did not have an effect on mortality, but rather delayed viral clearance. ${ }^{3}$

\section{$\underline{\text { Advice }}$}

As is current practice, injections must not be undertaken in individuals with active infections but the potential arises to do harm to individuals who may be incubating or later develop COVID-19.

Long acting, usually insoluble steroid formulations are frequently used in procedures to manage pain. To put this into context, Triamcinolone Acetonide $40 \mathrm{mg}$ is equivalent to ten times the normal daily physiological steroid production. Epidural steroids have been shown to cause a variable degree of adrenal suppression for at least some weeks. ${ }^{4}$ The potential impact of this immunological suppression in a patient incubating COVID at the time or in the future is unknown. 
As with all pain management procedures a risk benefit balance has to be reached in discussion with the patient. Each case is unique and no guidance will cover all eventualities.

Caution should be used when assessing patients for steroid injections balancing the severity of underlying disease, the potential benefit and the risks including immunocompromise, underlying comorbidities and further risk should the patient have or become infected in the period after injection when there is likely to be an ongoing effect on the immunological system.

Patients should be fully aware of the potential increased risk, the lack of clear evidence and be engaged in decision making. Likewise, individual units should consider the risk and benefits of such injections and under which circumstances they will continue using them during the current clinical conditions.

\section{The future}

The situation relating to the pandemic is rapidly changing but it is thought that there will be several months of disruption and the potential for a significant proportion of the population to become infected.

National guidance should also be followed and will be updated on a regular, often daily basis.

17 March 2020

1. Clinical management of severe acute respiratory infection when novel coronavirus (nCoV) infection is suspected. 2020 World Health Organization. WHO. https://www.who.int/docs/defaultsource/coronaviruse/clinical-management-of-novel-cov.pdf

2. Russell CD, Millar JE, BaillieJK. Clinical evidence does not support corticosteroid treatment for 2019-nCoV lung injury. Lancet 2020;395:473.

3. Huang et al, Clinical features of patients infected with 2019 novel coronavirus in Wuhan, China www.thelancet.com Vol 395 February 15, 2020

4. Friedly J et al, Systemic effects of epidural steroid injections for spinal stenosis, Pain: May 2018, Vol 159, Issue $5, \mathrm{p} 876-883$ 